\title{
A Cognitive Portrayal of Risk Perception in Turkey: Some Cross-national Comparisons
}

\begin{abstract}
Mary E. Thomson, Dilek Önkal and Gülbanu Güvenç ${ }^{l}$
This paper describes an exploratory investigation of Turkish risk perceptions of various activities and technologies. Factor analysis $r$ evealed various meaningful $r$ esults, including distinct locations in the T urkish cognitive map for nuclear power, large construction and commercial aviation risk items. The differences in the positioning of certain clusters of risk items within the Tirkish map are also notable, particularly the cluster of food preservatives, food colourings and pesticides. Such locations ae found to contrast considerably with those obser ved in risk perception studies relating to other nations, such as the USA and Japan. Possible explanations are offered for the findings, and potential avenues for future research are provided.
\end{abstract}

Key Words: Risk perception; psychometric paradigm; cross-national comparisons; cognitive map

\section{Introduction}

How ordinary people perceive various societal risks has been the subject of much academic attention in recent years. Risk perception refers to people's judgements and evaluations of hazards which they are or might be exposed to, and research in this context is important to academics for two main reasons (Renn and Rohrmann, 2000). The first reason relates to the communication of risks. Since risk communication is designed to arm ordinary people with the information they require to make informed decisions about risks to their health, safety and environment, such communication

cannot be effective without a comprehensive understanding of how people perceive and evaluate risks and why risk perception varies so much within a society. (Morgan, 2002:4)

Secondly, it is vital to know what concerns people and whyso that these views can be incorporated into important political decisions.

By far the most-utilised approach to the study of risk perception has been the psychometric paradigm, which originated in the work of the 'Oregon Groupin the 1980s. ${ }^{2}$ As defined recently by Paul Slovic, the psychometric approach

\footnotetext{
encompasses a theoretical framework that assumes risk is subjectively defined by individuals who may be influenced by a wide array of psychological, social, institutional and cultural factors ... [and] with appropriate design of survey instruments, many of these factors and their interrelationships can be quantified and modelled in order to illuminate the responses of individuals and societies to the hazards that confront them. (2000:xxiii)
}

Accordingly, the Oregon Group attempted to reveal peoples expressed preferences in relation to their perceived tolerability of various societal risks. They aimed to gain an insight into people's perceptions of the frequency and probability of risks, with a view to establishing the extent to 
which these perceptions are biased or contain inaccuracies, and to understanding the cognitive processes underlying such judgements. With the use of factor analysis, three basic dimensions in relation to public risk perceptions were identified (Fischhoff et al, 1978), including:

- dread risk, characterised by a 'perceived lack of control, dread, catastrophic potential, fatal consequences, and the inequitable distribution of risks and benefits' (Slovic, 1987:283);

- unknown risks, relating to judgements of the observability of risks, the degree of uncertainty or unfamiliarity, and whether or not the effects are delayed in time; and

- numbers exposed, addressing the number of people likely to be afected in terms of deaths, injuries or ill health if the risk event occurs.

The psychometric approach has been influential in terms of providing the groundwork for subsequent research, which has identified a finite range of core risk evaluation dimensions which, it is argued, influence people's perceptions of risk. Much research following on from that of the Oregon Group has suggested that perceived risk is quantifiable and predictable (for instance, Gardner et al, 1982; Johnson and Tversky, 1984; Lindell and Earle, 1983; Vlek and Stallen, 1981).

Given that risk perceptions were argued to be quantifiable, an increasing number of studies have focused on cultural/national differences that may exist in perceiving risks. This particular focus has attracted attention for various reasons. For one thing, it throws light on theenerality of basic findings from previous studies conducted in the Western world (Renn and Rohrmann, 2000). In addition, an appreciation and understanding of the main concerns and fears inherent in other cultures/nations can be used to good efect in the improvement of international relations.To date, however, most risk perception research has been directed towards European and NortAmerican countries (for instance, vonWinterfeldt and Edwards, 1984; Englanderet al, 1986; Mechitov and Reblik, 1990). A few studies have focused on countries such as China and Japan (for instance, Keown, 1989; Rosa et al, 2000), but developing countries have on the whole been ignored. Accordingly, the present study aims to take one step in addressing this research gap by providing an exploratory psychometric investigation of risk perceptions in a developing country with a unique cultural framework: Turkey. Turkey presents a challenging starting point for such analyses, since it is ar guably the most Westernised of Near-Eastern countries (Cindoglu, 1991), going through a transformation since the 1980s that involvesintegration with the world economy and a pending European Union membership candidacy (White, 2002; Günçavdı et al, 1998).

It must be acknowledged that the adoption of an exclusively psychometric approach suffrs from potential limitations, such as its failure to account for political and social manifestations (Douglas and Wildavsky, 1982) and the context-dependent nature of risk judgements (see, for example, Singleton and Hovden, 1987). However, given that the present study is designed to represent an initial exploratory cognitive investigation of Turkish risk perceptions, and given that the psychometric approach is typically used for cross-national comparisons, it is arguably ideal for the present purposes (see, for instance, Englander et al, 1986; Teigen et al, 1988; Keown, 1989; Mechitov and Reblik, 1990; Goszczynska et al, 1991).

A brief review of the cultural comparison literature is provided next. This is followed by an outline of the methodology used in the present study and a detailed analysis of the data. Finally the paper concludes with a discussion of the main findings in relation to those of other studies, and with suggestions for future research.

\section{Cultural comparisons}

Cultural differences in risk perception have been investigated from several perspectives, based on intra-national group comparisons or cross-national studies. In intra-national comparisons, researchers have explored cultural sub-groups within a countrycomparing the worldviews of the 
particular subgroups (for example, Borcherdinget al, 1986; Marris et al, 1996; Rohrmann, 1994). Such studies have utilised the 'cultural approach' (Douglas and Wildavsky, 1982), which takes the view that risk is a social and cultural construction and attempts to discover what dif ferent aspects of social life educe particular responses to danger.

Cross-national studies have tended to use the psychometric approach in various ways. While some researchers have conducted comparative research in two countries (for instance, Eisert al, 1990; Renn and Swaton, 1984), others have replicated previous US studies in diffent countries to enable comparisons with previously established results (Englander et al, 1986).

In a recent review of cultural comparisons in risk perception, Rohrmann and Renn (2000) discussed the findings of 20 such studies.A core finding that emerged from this review is that vast dferences are often apparent in judgements, depending on the social, political and economic emphasis in the countries under consideration. Quite disparate perceptions of nuclear war and smoking risks have been found among nations such as France, Sweden, GermanyChina, Japan and the US. For instance, in a study comparing Japan and the US the mean ratings for the Japanese tended to be slightly higher, although ranked in a similar way, for the various activities and technologies (Hinman et al, 1993). However, the perceived risk from nuclear ener gy and nuclear war was markedly higher than that of theirAmerican counterparts. Given that the Japanese had the horrific experience of suffering two nuclear attacks in the Second World War, it is easy to understand their accentuated nuclear-related concerns and fears.

Other cross-cultural findings have emerged from the work of Sjöberg et al (2000), who found risk perceptions in Bulgaria and Sweden to dif fer substantially on a wide variety of risks, including pregnancy and childbirth, crime, food irradiation, $\mathrm{x}$-rays, fertilisers and antibiotics. In relation to such risks, Bulgarian evaluations were dramatically higher than those of their Swedish counterparts, probably reflecting the relative political, social and economic conditions in these countries. Certainly Sweden is an affluent, knowledgeable, fully democratic welfare state, with high life expectancy and low levels of crime. Conversely, Bulgaria is undergoing transition from a single-party communist state where media information was strictly controlled by the government; in addition, it is economically and politically turbulent, with low living standardsAlso, the same authors found that some Romanian perceptions, such as those concerning asbestos, antibiotics and fertilisers, were far closer to those of the Swedes than they were to those of the Bulgarians, despite the similarities between Romania and Bulgaria in terms of geography and recent historyThe authors noted, however, that the Romanian media were more candid about domestic risks than their Bulgarian counterparts, while the latter provided more extensive coverage of risks in general at the time of the studyThey therefore suggested media content as a potential determining factor of the observed differences.

Another finding that emerged from the Sjöberg et al (2000) study related to gender differences. Large differences were evident between men and women in Romania, with women providing higher ratings for most of the risks. Such findings are relatively common (Barke et al, 1997; Savage, 1993) and are generally accounted for in terms of social roles, status diferentiation and biological differences. Sjöberg et al also found considerable occupational dif ferences in both Romania and Bulgaria, with the lowest ratings of perceived risk consistently coming from students.

\section{Research design}

The current research used the study conducted by Fischhof et al (1978) in the US, adapting it to Turkish respondents. The items finally used were pre-tested with a group of business students to identify any culture-specific factors, leading to an elimination of two items (American football and lawnmowers) out of the initial list of 30. The participants evaluated a total of 28 activities and technologies on various risk scales. These items are displayed in Table 1. A language expert translated the questionnaire used in the original study intoTurkish so that the respondents would have no difficulty in understanding the questions. Items were also back-translated to ensure that there remained no discrepancies in meaning stemming from translations. 
Table I. 28 Risk items

\begin{tabular}{ll}
\hline & Activity or technology \\
\hline 1 & Alcoholic beverages \\
2 & Bicycles \\
3 & Commercial aviation \\
4 & Contraceptives \\
5 & Electric power (non-nuclear) \\
6 & Fire fighting \\
7 & Food coloring \\
8 & Food preservatives \\
9 & General (private) aviation \\
10 & Handguns \\
11 & Home appliances \\
12 & Hunting \\
13 & Large construction (dams, bridges, etc) \\
14 & Motorcycles \\
15 & Motor vehicles \\
16 & Mountain-climbing \\
17 & Nuclear power \\
18 & Pesticides \\
19 & Police work \\
20 & Prescription antibiotics \\
21 & Railroads \\
22 & Skiing \\
23 & Smoking \\
24 & Spray cans \\
25 & Surgery \\
26 & Swimming \\
27 & Vaccinations \\
28 & X-rays \\
\hline
\end{tabular}

Table 2. Seven risk characteristics

\begin{tabular}{|c|c|c|}
\hline & Question & Scale \\
\hline 1. & $\begin{array}{l}\text { Voluntariness of risk: do people get into these risky situations } \\
\text { voluntarily? }\end{array}$ & 1: voluntary; 7 : involuntary \\
\hline 2. & $\begin{array}{l}\text { Immediacy of effect: to what extent is the risk of death } \\
\text { immediate or is death likely to occur at some time later? }\end{array}$ & 1: immediate; 7 : delayed \\
\hline 3. & $\begin{array}{l}\text { Knowledge about risk: to what extent are the risks known } \\
\text { precisely by the persons who are exposed to those risks? }\end{array}$ & 1: known precisely; 7: not known \\
\hline 4. & $\begin{array}{l}\text { Control over risk: if you are exposed to the risk of each } \\
\text { activity or technology, to what extent can you, by personal } \\
\text { skill or diligence, avoid death while engaging in the activity? }\end{array}$ & 1: uncontrollable; 7 : controllable \\
\hline 5. & Newness: are these risks new, novel ones or old, familiar ones. & 1: new; 7: old \\
\hline 6. & $\begin{array}{l}\text { Chronic-catastrophic: is this a risk that kills people one at a } \\
\text { time (chronic) or a risk that kills large numbers of people at one } \\
\text { time (catastrophic)? }\end{array}$ & 1: chronic; 7: catastrophic \\
\hline 7. & $\begin{array}{l}\text { Common-dread: is this a risk that people have learned to live } \\
\text { with and can think about reasonably calmly, or is it one that } \\
\text { people have great dread for? }\end{array}$ & 1: common; 7: dread \\
\hline
\end{tabular}

Note: the 28 activities and technologies were included on each scale (T able 1). 
The participants were required to rate each activity or technology on seven risk characteristics with regard to their perceived risk on seven-point scales (Table 2 ). ${ }^{3}$

\section{Sample}

Of the overall total of 150 questionnaires sent out to potential participants, 73 were returned. There were 32 female and 41 male respondents. The mean age of participants was 29 and the standard deviation was nine years (with an age range between 19 and 56). Of the returned questionnaires 23 were from management and engineering students from universities inAnkara; the remaining 50 were from Turkish project development professionals working on information system projects. This particular sample was sought in the present exploratory study in an £6rt to ensure that the respondents had at least a basic level of awareness of science and technology , given the risks under consideration.

\section{Results}

The data were first subjected to principal-components factor analysis. Varimax-rotated factorloadings across seven risk characteristics are shown inTable $3 .{ }^{4}$ This table indicates the degree to which each risk characteristic is correlated with each of the two underlying factors. Loadings below 0.45 are considered to indicate items explaining a poor percentage of variance and thus are not included in factor interpretations (Comrey, 1973). Accordingly, the first factor is found to be associated with the risk dimensions of common/dread, chronic/catastrophic and new/old, and to a lesser extent, 'control over risk'(uncontrollable/controllable). The second factor is found to be highly correlated with 'voluntariness of risk' ( voluntary/involuntary), 'knowledge about risk' (known precisely/not known) and 'immediacy of effect' (immediate/delayed).

Table 3. Factor loadings across seven risk characteristics

\begin{tabular}{|c|c|c|}
\hline & \multicolumn{2}{|c|}{ Component $^{*}$} \\
\hline & 1 & 2 \\
\hline Voluntariness & 0.00071 & 0.775 \\
\hline Immediacy & -0.336 & 0.646 \\
\hline Knowledge & 0.303 & 0.770 \\
\hline Control & -0.482 & 0.333 \\
\hline Newness & -0.520 & -0.011 \\
\hline Chronic-Catastrophic & 0.719 & 0.047 \\
\hline Common-Dread & 0.736 & 0.023 \\
\hline
\end{tabular}

Extraction method: principal component analysis.

Rotation method: varimax with Kaiser normalization.

* Rotation converged in three iterations.

Given that each of the 28 items had a mean score on each of the seven characteristics, a score for each item or each factor was obtained.These factor scores enabled a plot of 28 items in the space defined by the two factors. Figure 1 displays a plot of these 28 items. 
Figure I. Location of risk items within the two-factor space

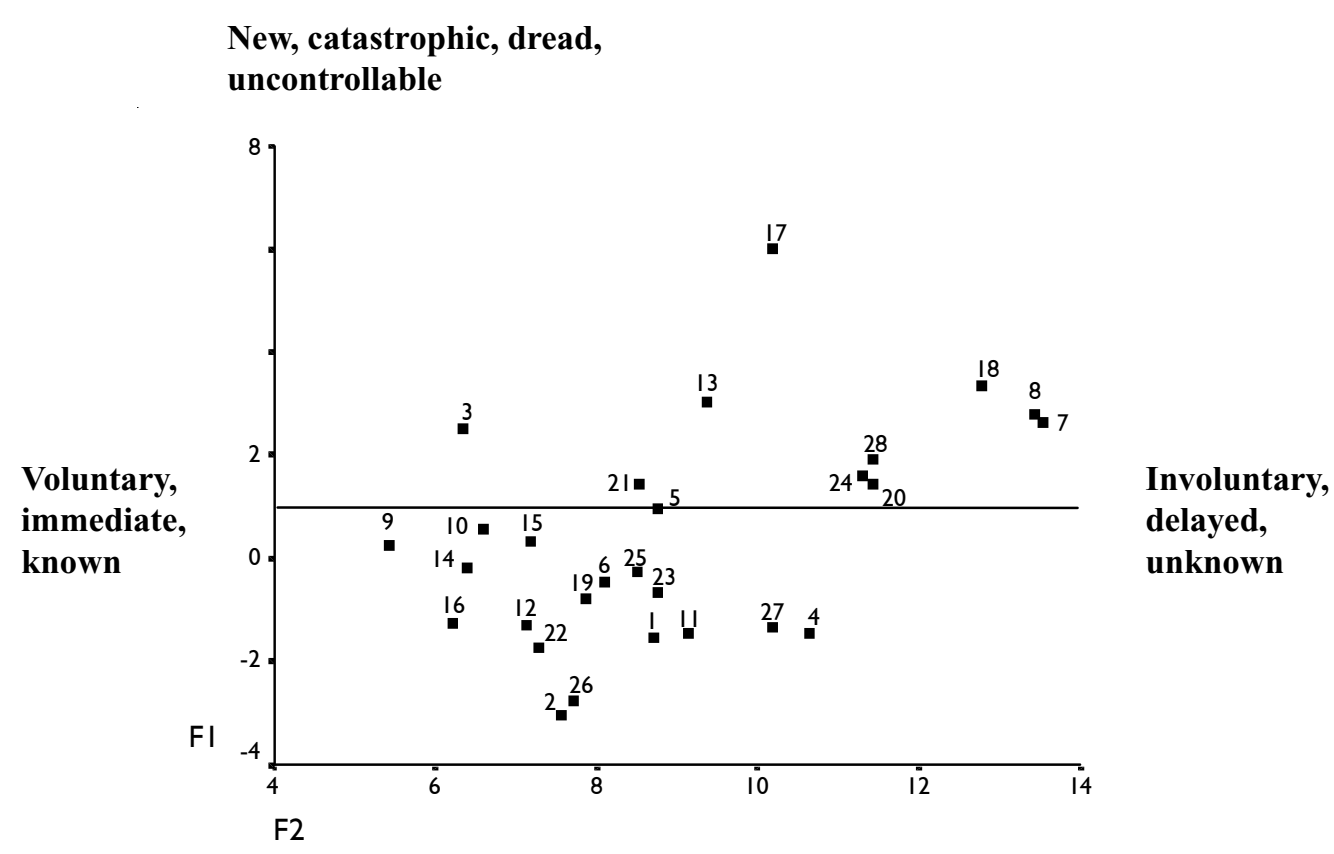

Old, chronic, common, controllable

This plot helps to clarify the nature of the two factorsThe upper extreme of factor 1 is associated with perceptions of dreaded, catastrophic, new and uncontrollable items. Items low on the first factor are familiar, controllable activities with consequences perceived at the individual level. However, higher scores on factor 2 are associated withnot known and involuntary technological risks, and events whose consequences are to be realised sometime later.

The position of nuclear power (item 17) within the factor space is of interest. Clearly , the participants in this study viewed the risks from nuclear power as qualitatively dif ferent from those of the other activities. Figure 2 highlights this dif ference by comparing the mean risk ratings for nuclear power and electric power (defined in the questionnaire as power not generated from nuclear sources). Nuclear power is perceived as markedly moreatastrophic, dreaded, new and uncontrollable, in comparison to electric power. It is worth noting here that specific nonnuclear sources such as wind, solar and hydro technologies have not been individually included in the items given to participants. Future extensions of this work could compare risk perceptions regarding such energy sources so as to provide policy guidance.

Large construction (item 13) is found to be one of the most dreaded items, and it remainsgnitively distinct from the others. One plausible explanation could be related to the mass destruction in the recent Turkish earthquakes, where the loss of thousands of lives was, to a significant extent, the product of shoddy building construction. Of course, risks associated with lage construction are also salient in the aftermath of the events of 1th September 2001, and it is notable that commercial aviation (item 3 ) is also a distinct item of roughly equivalent dread, only perceived to be more voluntary, immediate and known.

The close proximity of food colourings (item 7), food preservatives (item 8) and pesticides (item 18) demonstrates that this cluster overall is viewed as a not known, involuntary risk group whose consequences would occur sometime later. The similar ratings for food colourings and food preservatives suggest that the respondents did not appear to be aware of thdifferences between them. 
Figure 2. Qualititative characteristics of perceived risk for nuclear power and electric power

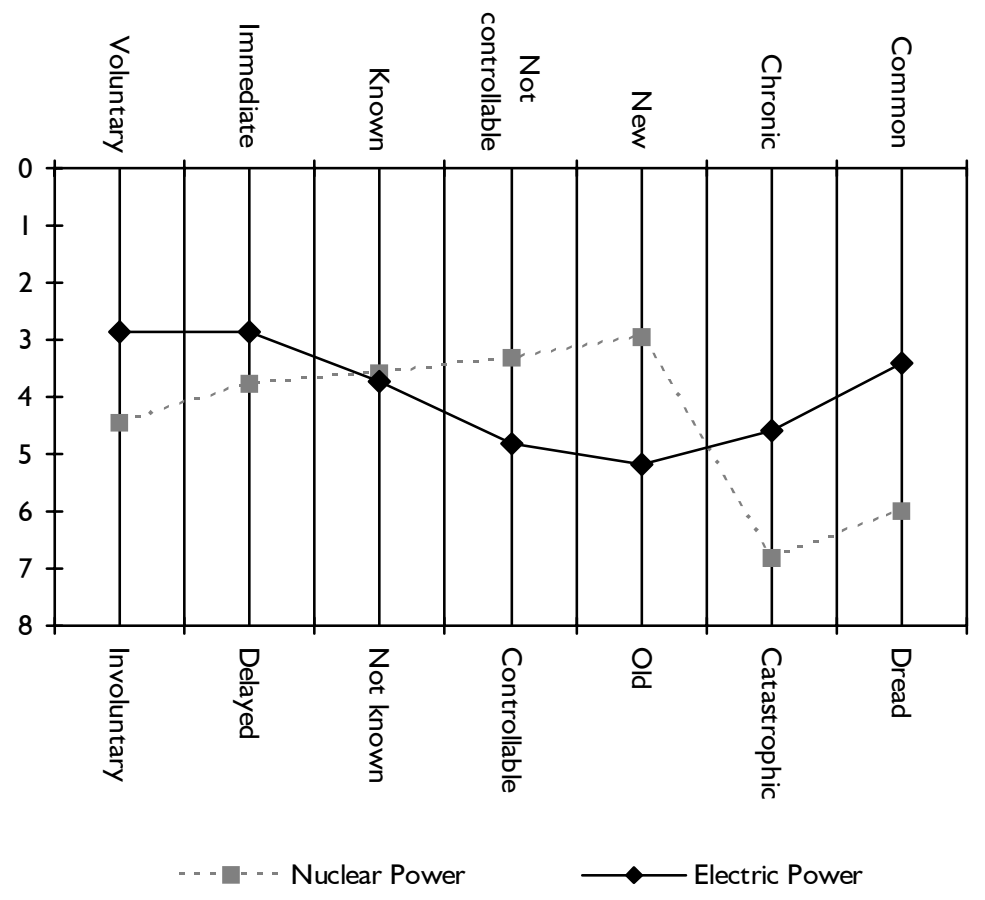

Antibiotics (item 20), spray cans (item 24) and X-rays (item 28) were also rated similarly by the respondents. These three are located close to thenot known side of Factor 2 in Figure 1, suggesting that knowledge of these risks may also be quite limited.

An examination of gender and occupational dif ferences in mean ratings for the various risk dimensions was conducted via independent samplest-tests. An analysis of gender effects reveals that gender difference is significant for the common/dread risk characteristic, as demonstrated in Table 4. In particular, female respondents appear to perceive risk items as being more dreaded ( $p$ $=.010$ ) than do male respondents. Occupational differences between students and professionals show no significant disparities for any of the risk dimensions (alp $>.10)$, suggesting the relative homogeneity of this particular sample's risk perceptions.

Table 4. Gender differences in mean ratings for the given risk dimensions

\begin{tabular}{lcccc}
\hline & $\begin{array}{c}\text { Male } \\
\text { participants }\end{array}$ & $\begin{array}{c}\text { Female } \\
\text { participants }\end{array}$ & $\boldsymbol{t}$-statistic & $\boldsymbol{p}$-value \\
\hline Voluntariness & 3.111 & 3.033 & .26 & .398 \\
Immediacy & 3.752 & 3.566 & .48 & .317 \\
Knowledge & 3.393 & 3.212 & .68 & .251 \\
Control & 4.312 & 4.271 & .21 & .416 \\
Newness & 5.031 & 4.773 & .88 & .192 \\
Chronic-Catastrophic & 3.693 & 3.897 & -.48 & .317 \\
Common-Dread & 3.117 & 3.702 & -2.38 & .010 \\
\hline
\end{tabular}




\section{Conclusions}

When the findings of the present study are compared to those of similar studies concerning other cultures, it is immediately clear that nuclear risk evokes strong perceptions of dread everywhere. As with the original study conducted by Fischhof f et al (1978) in the US, and others concerning Sweden, Bulgaria and Romania (Sjöbeg et al, 2000), for example, the present Turkish sample perceived nuclear risk to beunknown, new and uncontrollable, and qualitatively different from any of the other technologies and activities. In common with these nations, the present findings regarding nuclear risk contrasts with those observed in Hinmanet al's (1993) Japanese study in terms of the respondents' perception of such risks as being known precisely and old. As discussed above, the possible reasons for this difference are sadly obvious.

Although some potential explanations were discussed above, it is worth noting that the locations of large construction and commercial aviation contrast considerably with the findings in Fischhoff et al's (1978) original US study, in which the respondents' mean ratings for these risks were relatively low and were perceived as equivalent to the risks associated with riding a bicycle. Even though most of the previous risk perception research has been conducted in the US, this particular contrast highlights the need for further detailed investigation there given that the horrific events of 11th September may have significantly altered the structure of such perceptions.

Another finding highlighted in the current study is that of perceptions about risks relating to food colourings, food preservatives and pesticides. These risks were perceived by the Turkish respondents as involuntary, delayed and unknown. This contrasts sharply with a recent study comparing the US and Japan (Rosa et al, 2000), in which respondents perceived such risks to be old and known; while it is similar to the findings on these items in Fischhoff et al's (1978) earlier USA study. Given the importance attached to food labelling in the US (especially in the period following the work of Fischhof $\mathrm{f}$ and his colleagues) and the associated activities of consumer-protection movements, these results may simply reflect a current lack of appropriate information about such risks in the developing country of Turkey, as was assumed to be the case in Bulgaria (Sjöberg et al, 2000).

Similarly, X-rays, antibiotics and spray cans were closely related items ifTurkish factor space. These items have also been found to be closely located in a variety of other studies (Sjöbgret $a l, 2000)$, including those concerning the recent US/Japan comparison. Howeveran important difference in the present study is that these items were rated as more unknown and the risks involuntary than in the other studies. It is also notable that X-rays and antibiotics were perceived above average in terms of dread in the present study. Again, this is similar to how they were perceived by Bulgarians.

Current work has also found that gender appears to have a significant ef fect on the dread dimension. As emphasised previously, this is a relatively common finding. Women tend to perceive greater risk than men do, even though they are statistically less exposed to danger than are men (Savage, 1993). One implication of such findings is that producers of technologically hazardous products that are primarily consumed by women should realise that women may view their products with considerably more scepticism.

The present study is instrumental in revealing crucial insights about Turkish perceptions of risk and how they compare to those of other nations. The most compelling feature of the Turkish cognitive map relates to the distinct locations of the nuclear large construction and commercial aviation items. In addition, the differences in the positioning of certain clusters of risk items within the map are notable. The most salient of these is the cluster of food 
preservatives, food colourings and pesticides. When the current findings are compared to those of other nations, it is clear thatTurkish people often dread some of the same potentially catastrophic events, but arrive at this common point via dif ferent cognitive routes (eg as evidenced in the ratings of the X-rays, antibiotics and spray cans items, which were perceived to be more unknown and involuntary than in most other studies). Some of the highly dreaded Turkish items, however, were quite unique, and may have been a function of recent catastrophic events (eg earthquakes), either in Turkey or elsewhere. Accordingly, it is clear that further research is needed, both in Turkey and in other nations, to advance theoretical knowledge in this field and to aid in assisting the practical utilisation of the findings. Future investigations should utilise multiple paradigms to unravel the complex and tangled factors that influence human perceptions of risk.

\section{Notes}

Mary E. Thomson is a Reader in the Department of Psychology, Glasgow Caledonian University; email: mwi@gcuexch.gcal.ac.uk. Dilek Önkal is an Associate Professor of Decision Sciences and Associate Dean in the Faculty of Business Administration, Bilkent University, Ankara 06533; email: onkal@bilkent.edu.tr. Gülbanu Güvenç is a PhD student in Decision Sciences in the Faculty of Business Administration, Bilkent University; email: gulbanui@bilkent.edu.tr. White, Howard Kunreuther, Sarah Lichtenstein and Baruch Fischhoff.

It should be noted that the dimension of 'knowledge about risk' (to what extent the risks are known to science) and that of 'severity of consequences' (when the risk from the activity is realized, how likely it is that the consequence will be fatal) in Fischhof f et al's (1978) study were not included in the questionnaires, since initial interviews with participants indicated that these were considered relatively less important items given the other seven dimensions. Participants felt their inclusion would considerably increase the time to complete the task and would increase the effects of fatigue, reducing the motivation; they were thus excluded in the current study. Following similar arguments, Rosa et al (2000) have also used seven dimensions in their work with the Japanese risk perceptions.

A similar loading structure is found when an oblique rotation is performed. We do not report the detailed results of an oblique rotation since the correlations (among the factors) above 0.32 are accepted as suggesting that the oblique rotation should be preferred (itbachnick and Fidell, 1996), whereas our results yield a correlation of 0.075 .

\section{References}

Burke, R.P., Jenkins-Smith, H. and Slovic, P. (1997) Risk Perception of Men andWomen Scientists. Social Science Quarterly. Vol. 78, No. 1, pp 167-76.

Borcherding, K., Rohrmann, B. and Eppel, T. (1986) A Psychological Study on the Cognitive Structure of Risk Evaluations. In Brehmer, B., Jungermann, H., Lourens, P . and Sevon, G. (eds) New Directions in Research on Decision Making. Amsterdam: North-Holland.

Cindoglu, D. (1991) Re-Viewing Women: Images of Patriar chy and Power in Modern T urkish Film. Unpublished $\mathrm{PhD}$ dissertation, State University of New York at Buffalo.

Comrey, A.L. (1973) A First Course in Factor Analysis. New York: Academic Press.

Douglas, M. and Wildavsky, A. (1982) Risk and Culture: An Essay on the Selection of T echnical and Environmental Dangers. Berkeley, CA: University of California Press.

Eiser, J.R., Hannover, B., Mann, L. and Webley, P. (1990) Nuclear Attitudes after Chernobyl: A Cross National Study. Journal of Environmental Psychology. Vol. 10, No. 2, pp 101-10. 
Englander, T., Farago, K., Slovic, P. and Fischhoff, B. (1986) A Comparative Analysis of Risk Perception in Hungary and the United States. Journal of Social Behavior. Vol. 1, pp 55-66.

Fischhoff, B., Slovic, P., Lichtenstein S. and Combs, B. (1978) How Safe is Safe Enough? A Psychometric Study of Attitudes Toward Technological Risk and Benefits. Policy Sciences. Vol. 8, pp 127-52.

Gardner, G.T., Tierman, E.R., Gould, L.C., DeLuca, E.R., Doob, L.W. and Stolwijk, J.A.J. (1982) Risk and Benefit Perceptions, Acceptability Judgments and Self-Reported Actions Toward Nuclear Power.Journal of Social Psychology. Vol. 116, pp 179-97.

Goszczynska, M., Tyszka, T. and Slovic, P. (1991) Risk Perception in Poland: A Comparison with Three other Countries Journal of Behavioral Decision Making. Vol. 4, No. 3, pp 179-93.

Günçavdı, Ö., Bleaney, M. and McKay, A. (1998) Financial Liberalisation and Private Investment: Evidence from Turkey. Journal of Development Economics. Vol. 57, No. 2, pp 443-55.

Hinman, G.W., Rosa, E.A., Kleinhesselink, R.R. and Lowinger , T.C. (1993) Perception of Nuclear and Other Risks in Japan and the United States. Risk Analysis. Vol. 13, No. 4, pp 449-56.

Johnson, E.J. and Tversky, J. (1984) Representations and Perceptions of Risks. Journal of Experimental Psychology: General. Vol. 113, pp 55-70.

Keown, C.F. (1989) Risk Perception of Hong Kongese versus Americans. Risk Analysis. Vol. 9, No. 3, pp 401-7.

Lindell, M.K. and Earle, T.C. (1983) How Close is Close Enough? Public Perceptions of the Risks of the Risks of Industrial Facilities. Risk Analysis. Vol. 3, No. 4, pp 245-54.

Marris, C., Langford, I. and O'Riordan, T. (1996) Integrating Social and Psychological Approaches to Public Perceptions on Environmental Risks: Detailed Results fr om a Questionnaire Survey. Norwich: University of East Anglia.

Mechitov, A.I. and Reblik, S.B. (1990) Studies of Risk and Safety Perception in the USSR. In Borcherding, K., Larichev, O.J. and Mesick, D.M. (eds) Contemporary Issues in Decision Making. Amsterdam: NorthHolland.

Morgan, M.G. (2002) A Mental Approach. Cambridge: Cambridge University Press.

Renn, O. and Rohrmann, B. (eds) (2000) Cross-Cultural Risk Perception. Dordrecht: Kluwer Academic.

Renn, O. and Swaton, E. (1984) Psychological and Sociological Approaches to Study Risk Perception. Environment International. Vol. 10, pp 557-75.

Rohrmann, B. (1994) Risk Perception of Diferent Societal Groups: Australian Findings and Cross-National Comparisons. Australian Journal of Psychology. Vol. 46, pp 150-63.

Rohrmann, B. and Renn, O. (2000) Risk Perception Research:An Introduction. In Renn and Rohrmann, op cit.

Rosa, E.A., Matsuda, N. and Kleinhesselink, R.R. (2000) The Cognitive Architecture of Risk: Pancultural Unity or Cultural Shaping? In Renn and Rohrmann, op cit.

Savage, I. (1993) Demographic Influences on Risk Perceptions. Risk Analysis. Vol. 13, No. 4, pp 413-20. Singleton, W.T. and Hovden, J. (1987) Risk and Decisions. Chichester: Wiley.

Sjöberg, L., Kolarova, D., Rucai,A.A. and Bernström, M.L. (2000) Risk Perception in Bulgaria and Romania. In Renn and Rohrmann, op cit.

Slovic, P. (1987) Perception of Risk. Science. Vol. 236, pp 280-5.

Slovic, P. (2000) The Perception of Risk. London: Earthscan.

Tabachnik, B.G. and Fidell, L.S. (1996) Using Multivariate Statistics. 3rd edn. New York: Harper Collins.

Teigen, K.H., Brun, W. and Slovic, P. (1988) Societal Risks as Seen by a Norwegian Public. Journal of Behavioral Decision Making. Vol. 1, No. 1, pp 111-30. 
Vlek, C.A.J. and Stallen, P.J. (1981) Judging Risks and Benefits in the Small and in the Lge. Organizational Behavior and Human Performance. Vol. 28, pp 235-71.

White, J.B. (2002) The Islamist Paradox. In Kandiyoti, D. and Saktanber, A. (eds) Fragments of Culture: The Everyday of Modern Turkey. London: I.B.Tauris.

von Winterfeldt, D. and Edwards, W. (1984) Patterns of Conflicts about RiskyTechnologies. Risk Analysis. Vol. 4, No. 1, pp 55-68. 